\title{
Gestión del Conocimiento en la Educación
}

\author{
Knowledge Management in Education
}

Yira Muñoz-Sánchez ${ }^{a}$, María A. Alonso-Lavernia ${ }^{b}$, Iliana Castillo-Pérez ${ }^{b}$, Verónica Martínez-Lazcano ${ }^{b}$

\begin{abstract}
:
Knowledge management in education has become an essential element in today's societies, being considered by some as a discipline that allows improving teaching and learning processes for the acquisition of competences for the benefit of the comprehensive training of students and that It allows them to be competent and have a relevant impact, through innovation, scientific research and technology, on the competitiveness of the workplace.
\end{abstract}

Keywords:

Knowledge management, education, innovation, competence

Resumen:

La gestión del conocimiento en la educación se ha convertido en un elemento esencial en las sociedades actuales, siendo considerada por algunos como una disciplina que permite mejorar los procesos de enseñanza y aprendizaje para adquisición de competencias en beneficio de la formación integral de los estudiantes y que les permita ser competentes e incidir de manera relevante, a través de la innovación, la investigación científica y la tecnología, en la competitividad del ámbito laboral.

Palabras Clave:

Gestión del conocimiento, educación, innovación, competencia

\section{Introducción}

En los tiempos actuales, se ha demostrado que la gestión del conocimiento en un elemento fundamental dentro de las instituciones de educación en beneficio de la calidad en la producción científica y la innovación que se debe generar en éstas. La gestión del conocimiento también ha sido considerada por las empresas en su intento por ser más competitivas dentro de las nuevas economías y de la sociedad de la información, por lo que es necesaria la gestión del conocimiento en la educación al ser esta protagonista en el desarrollo económico y social de cualquier país.

\footnotetext{
a Autor de Correspondencia, Universidad Autónoma del Estado de Hidalgo, Escuela Superior de Ciudad Sahagún, https://orcid.org/00000002-4876-2747, Email: yira@uaeh.edu.mx

b Universidad Autónoma del Estado de Hidalgo, Instituto de Ciencias Básicas e Ingeniería, https://orcid.org/0000-0002-9839-8250, Email: marial@uaeh.edu.mx

c Universidad Autónoma del Estado de Hidalgo, Instituto de Ciencias Básicas e Ingeniería, https://orcid.org/0000-0002-8130-9231, Email: ilianac@uaeh.edu.mx

d Universidad Autónoma del Estado de Hidalgo, Instituto de Ciencias Básicas e Ingeniería, https://orcid.org/0000-0003-2172-4000, Email: vlazcano@uaeh.edu.mx
} 


\section{Descripción}

La gestión del conocimiento se ha convertido en un elemento fundamental para que las personas, las sociedades y las instituciones logren el éxito. Específicamente dentro de la educación permite transformar el conocimiento y ponerlo a disposición de las personas para generar sabiduría colectiva, innovación y poblaciones educadas que aprovechen las innovaciones de la ciencia y la tecnología y entonces constituir sociedades basadas en conocimiento, por lo cual debe formar parte de los procesos de enseñanza y aprendizaje. Para generar oportunidades de competitividad en los estudiantes y proporcionarles una formación integral es necesaria también la gestión del conocimiento, ya que es una opción idónea para el desarrollo de procesos innovadores en el desarrollo de competencias de una forma más eficiente y efectiva. La gestión del conocimiento dentro de las universidades se mide a través de las publicaciones científicas y los trabajos de tesis que desarrollan en conjunto los profesores con los estudiantes.

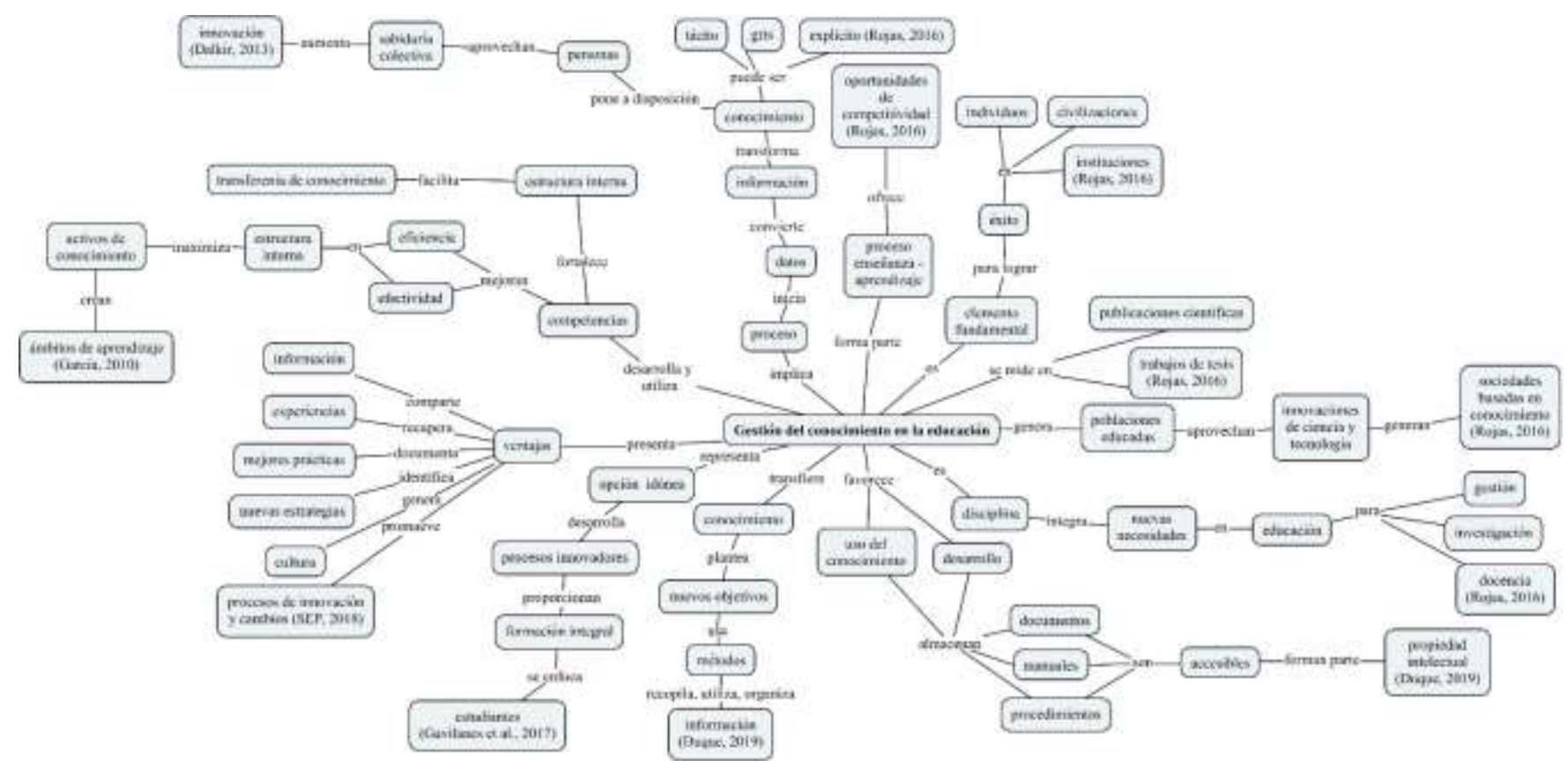

Figura 1. Gestión del conocimiento en la educación

\section{Referencias}

Dalkir, K. (2013). Knowledge management in theory and practice. Chapter 1. Estados Unidos: Routledge.

Duque, A. P. G., Contreras, D. O., \& García, E. M. M. (2019). Las competencias científicas a partir de la gestión del conocimiento en Instituciones de Educación Superior. Signos: Investigación en sistemas de gestión, 11(2), 21-40.

García, J. F. (2010). Gestión de la información y el conocimiento. México: Universidad de Guadalajara.

Gavilanes, J. G. P., Andrade, D. M. C., Samaniego, V. M. V., \& Román, J. Á. (2017). La Gestión del conocimiento y su pertinencia de en la Educación Universitaria. Revista Científica Hermes, 19, 475-493.

Rojas, A. E. (2016). Gestión del conocimiento en la educación universitaria. Población y Desarrollo, 19(35), 79-93.

SEP (2018). Marco Conceptual sobre Gestión del Conocimiento. Recuperado de https://usp.funcionpublica.gob.mx/html/DocumentacionDGDHSPC/AutogestionConocimiento/MarcoConceptual_GestionConocimiento.pdf 